\title{
CONSTRAINING THE GRB-MAGNETAR MODEL BY MEANS OF THE GALACTIC PULSAR POPULATION
}

\author{
N. Rea ${ }^{1,2}$, M. Gullón ${ }^{3}$, J. A. Pons ${ }^{3}$, R. Perna ${ }^{4}$, M. G. Dainotti ${ }^{5,6,7}$, J. A. Miralles ${ }^{3}$, And D. F. Torres ${ }^{2,8}$ \\ ${ }^{1}$ Anton Pannekoek Institute for Astronomy, University of Amsterdam, Postbus 94249, NL-1090 GE Amsterdam, The Netherlands \\ ${ }^{2}$ Instituto de Ciencias de l'Espacio (ICE, CSIC-IEEC), Campus UAB, Carrer Can Magrans s/n, E-08193 Barcelona, Spain \\ ${ }_{4}^{3}$ Departament de Fisica Aplicada, Universitat d'Alacant, Ap. Correus 99, E-03080 Alacant, Spain \\ ${ }^{4}$ Department of Physics and Astronomy, Stony Brook University, Stony Brook, NY 11794, USA \\ ${ }^{5}$ Physics Department, Stanford University, Via Pueblo Mall 382, Stanford, CA, USA \\ ${ }^{6}$ INAF-IASF Bologna, via P. Gobetti 101, 40129 Bologna, Italy \\ ${ }^{7}$ Obserwatorium Astronomiczne, Uniwersytet Jagielloński, ul. Orla 171, 31-501 Kraków, Poland \\ ${ }^{8}$ Institució Catalana de Recerca i Estudis Avanats (ICREA), E-08010 Barcelona, Spain \\ Received 2015 July 29; accepted 2015 October 5; published 2015 November 3
}

\begin{abstract}
A large fraction of Gamma-ray bursts (GRBs) displays an X-ray plateau phase within $<10^{5} \mathrm{~s}$ from the prompt emission, proposed to be powered by the spin-down energy of a rapidly spinning newly born magnetar. In this work we use the properties of the Galactic neutron star population to constrain the GRB-magnetar scenario. We reanalyze the X-ray plateaus of all Swift GRBs with known redshift, between 2005 January and 2014 August. From the derived initial magnetic field distribution for the possible magnetars left behind by the GRBs, we study the evolution and properties of a simulated GRB-magnetar population using numerical simulations of magnetic field evolution, coupled with Monte Carlo simulations of Pulsar Population Synthesis in our Galaxy. We find that if the GRB X-ray plateaus are powered by the rotational energy of a newly formed magnetar, the current observational properties of the Galactic magnetar population are not compatible with being formed within the GRB scenario (regardless of the GRB type or rate at $z=0$ ). Direct consequences would be that we should allow the existence of magnetars and "super-magnetars" having different progenitors, and that Type Ib/c SNe related to Long GRBs form systematically neutron stars with higher initial magnetic fields. We put an upper limit of $\leqslant 16$ "super-magnetars" formed by a GRB in our Galaxy in the past Myr (at 99\% c.1.). This limit is somewhat smaller than what is roughly expected from Long GRB rates, although the very large uncertainties do not allow us to draw strong conclusion in this respect.
\end{abstract}

Key words: gamma-ray burst: general - pulsars: general - stars: magnetars

\section{INTRODUCTION}

Gamma-ray bursts (GRBs) are one of the most extreme and powerful transient phenomena in the universe. They are generally divided in two groups, which have been proposed to have two distinctly different origins: Long GRBs (LGRBs), connected to the Type $\mathrm{Ib} / \mathrm{c}$ Core-Collapse Supernovae, and Short GRBs (SGRBs), originating from the merger of two neutron stars or a neutron star and a black hole.

Independently of the progenitor scenario, the prompt $\gamma$-ray emission is followed by intense longer-wavelength emission (afterglow). According to the standard "Fireball" theory, this radiation arises from the formation of a blast wave, due to a relativistic outflow pushing through the interstellar medium (Mészáros \& Rees 1997; Sari et al. 1998). In the past decade, thanks to Swift, the sample of Long and Short GRBs with a good multi-band monitoring of the afterglow became sufficiently large to enable a statistical study of the afterglow characteristics and energetics (Nousek et al. 2006; O'Brien et al. 2006; Zhang et al. 2006; Willingale et al. 2007; Evans et al. 2009; Dainotti et al. 2011b; Margutti et al. 2013; Dainotti et al. 2015b). It was observed that most GRBs do not show a smooth decay in X-ray flux after the prompt emission, as expected for a pure fireball model, but present rather ubiquitous $\mathrm{X}$-ray plateaus at times $<10^{5} \mathrm{~s}$, eventually pointing to a continuos energy injection in the first hours/day after the GRBs. These X-ray plateaus are generally interpreted as due to: a newly born rapidly spinning magnetar (see i.e., Metzger et al. 2011), an accreting black hole (see i.e., Kumar et al. 2008) or a top-heavy jet evolution (Duffell \&
MacFadyen 2015). The similarity of these plateau phases between the two classes of GRBs was ascribed to a common injection scenario. The fluence of these plateaus in both LGRBs and SGRBs is comparable with that of the prompt emission (never lower than an order of magnitude), and their luminosities and durations are observed to be anti-correlated (Dainotti et al. 2008, 2010, 2011a, 2013b; Rowlinson et al. 2013, 2014).

The latter correlation, combined with the fact that a newly born magnetar could be formed either via the collapse of a massive star (hence via a LGRB), or during the merger of two neutron stars (hence via a SGRB), motivated the interpretation of these X-ray plateaus as resulting from the delayed injection of rotational energy (with $\dot{E}_{\text {rot }} \sim 10^{50}-10^{51} \mathrm{erg} \mathrm{s}^{-1}$ ) from a fast spinning magnetar (Usov 1992; Zhang \& Mészáros 2001; Metzger et al. 2011).

Further support to the GRB-magnetar scenario was provided by the successful fitting of a large sample of Long and Short GRB afterglows (Zhang \& Mészáros 2001; Troja et al. 2007; Lyons et al. 2010; Rowlinson et al. 2010, 2013; Dall'Osso et al. 2011; Bernardini et al. 2012; Lü \& Zhang 2014) by modeling the plateau duration and luminosity in terms of the spin-down energy release timescale $\left(\tau_{\mathrm{sd}}\right)$ and luminosity $\left(L_{\mathrm{sd}}\right)$ :

$$
\begin{aligned}
T_{3} & \simeq \tau_{\mathrm{sd}} \\
= & 2.05\left(I_{45} B_{p, 15}^{-2} P_{\mathrm{ms}}^{2} R_{6}^{-6}\right) \\
L_{49} \simeq L_{\mathrm{sd}} & =\left(B_{p, 15}^{2} P_{\mathrm{ms}}^{-4} R_{6}^{6}\right),
\end{aligned}
$$

where $T_{3}$ is the plateau duration in $10^{3} \mathrm{~s}, L_{49}$ is the plateau luminosity in $10^{49} \mathrm{erg} \mathrm{s}^{-1}, I_{45}$ is the moment of inertia in units 
of $10^{45} \mathrm{~g} \mathrm{~cm}^{2}, B_{p, 15}$ is the magnetic field strength at the poles in units of $10^{15} \mathrm{G}, R_{6}$ is the radius of the neutron star in $10^{6} \mathrm{~cm}$, and $P_{\mathrm{ms}}$ is the initial period of the pulsar in milliseconds (Zhang \& Mészáros 2001; Metzger et al. 2011). ${ }^{8}$ In this scenario, the spin down luminosity and duration are expected to be anti-correlated as: $\log \left(L_{\mathrm{sd}}\right)=a-\log \left(\tau_{\mathrm{sd}}\right)$, where $a=\log \left(10^{52} I_{45}^{-1} P_{-3}^{-2}\right)$. Fitting the intrinsic plateaus it has been obtained that $a=52.7 \pm 0.5$ and $L_{49} \propto T_{3}^{(-1.07 \pm 0.14)}$ (Dainotti et al. 2013b; Rowlinson et al. 2014).

In our Galaxy we have discovered in the past few decades about 20 magnetars (Duncan \& Thompson 1992; see Mereghetti 2008; Rea \& Esposito 2011 for recent reviews, and the McGill Magnetar Catalog $\left.{ }^{9}\right)$. They are characterized by relatively bright $\mathrm{X}$-ray luminosities $\left(L_{\mathrm{X}} \sim 10^{33}-10^{35} \mathrm{erg} \mathrm{s}^{-1}\right)$, rotational periods in the $0.3-12 \mathrm{~s}$ range, strong $\mathrm{X} / \gamma$-ray flares and outburst activity, dipolar magnetic fields in the $6 \times 10^{12}$ $10^{15} \mathrm{G}$, and estimated ages between $\sim 1-10^{3} \mathrm{kyr}$.

In this paper we investigate the possible GRB origin of the magnetars in our Galaxy, as well as derive the limits on the GRB-magnetar scenario imposed by the properties of the Galactic magnetars. In Section 2 we re-analyze the Swift data of GRBs with good redshift measurements. Fitting them with the GRB-magnetar model (see also Rowlinson et al. 2014), we derive initial magnetic fields and spin period distributions for the sample. In Section 3 we use state-of-the-art magnetar evolution models (Viganò et al. 2013) coupled with Pulsar Population Synthesis simulations (Gullón et al. 2014, 2015) to constrain the current properties of possible magnetars formed via a GRB in our Galaxy in the past Myr, by comparing synthetic populations with the observed Galactic population. In Section 4 we discuss our results as well as the issue of how many and which GRBs are expected to leave behind a longlived stable magnetar, and the large uncertainties in the local GRB rates. We summarize our results and draw conclusions in Section 5 .

\section{FITTING MAGNETAR-DRIVEN PLATEAUS TO THE SWIFT LIGHT CURVES}

We re-analyzed the sample of all GRB X-ray afterglows, detected by Swift from 2005 January up to 2014 August with firm redshift measurements, and for which the light curves include early X-ray data and can be fitted by the Willingale et al. (2007) phenomenological model. We followed the fitting procedure presented in Dainotti et al. (2013b), and we use the redshifts available in the literature (Xiao \& Schaefer 2009), in the Greiner web page ${ }^{10}$ and in the Circulars Notice archive (GCN). The total number of GRBs with known redshift (in the 0.033-9.4 range) observed by Swift until 2014 August 14th is 283 (63 of which are SGRBs, and 25 are X-ray Flashes; XRFs), but not all these GRBs show a well-defined X-ray plateau emission. We found that among those, $176 \mathrm{GRBs}$ afterglows (14 of which are SGRBs, and all the 25 XRFs) are well fitted by an X-ray plateau model, as described in Dainotti et al. (2013b). The fitting procedure fails either when it gives

\footnotetext{
8 These equations apply to the electromagnetic dominated spin down regime, since the gravitational wave dominated regime would be extremely rapid and produce a negligible electromagnetic signal. It is also assumed that the loss of rotational energy is given by the magneto-dipole formula, whose validity in this scenario is highly questionable.

9 http://www.physics.mcgill.ca/ pulsar/magnetar/

10 http://www.mpe.mpg.de/ jcg/grbgen.html
}

unreasonable values of the errors or when the determination of confidence interval in $1 \sigma$ does not fulfill the Avni (1976) prescriptions. $^{11}$

We plot in Figure 1 (left panel) the rest-frame plateau luminosities in the Swift bolometric band $\left(E_{\min }=1\right.$, $E_{\max }=10,000 \mathrm{keV}$ ) at the time $T_{a}$ (end time of the plateau). The luminosities are computed from: $L_{X}\left(E_{\min }, E_{\max }, T_{a}\right)=$ $4 \pi D_{L}^{2}(z) F_{X}\left(E_{\min }, E_{\max }, T_{a}\right) \times K$, where $D_{L}(z)$ is the GRB luminosity distance (we have assumed a $\Lambda \mathrm{CDM}$ flat cosmological model with $\Omega_{M}=0.28$ and $\left.H_{0}=70 \mathrm{~km} \mathrm{~s}^{-1} \mathrm{Mpc}^{-1}\right), F_{X}$ is the measured X-ray energy flux, $K=(1+z)^{-1+\beta_{a}}$ is the so called $K$-correction, where $\beta_{a}$ is the spectral index assuming a simple power law spectrum (Evans et al. 2009; Dainotti et al. 2010). Note that, in the current paper we use the variables $L_{49}$ and $T_{3}$ corrected for selection bias and redshift evolution (namely, de-evolved). This approach is slightly different from the one presented in Rowlinson et al. (2014): in this work we derive the slope of the correlation directly by using the deevolved luminosity and time observables. In Rowlinson et al. (2014) the slope is fixed to the intrinsic one and the normalization is derived from the simulated data. This slightly different approach do not change the results, since the intrinsic slope used in Rowlinson et al. (2014) has been computed taking into account the same evolution. Caveats on the use of the observed slope instead of the intrinsic one have been discussed in Dainotti et al. (2013a). Since the rest frame time and luminosity we use are already corrected for selection bias and redshift evolution, the derived spin period and the magnetic field are unbiased too (this is a crucial point often omitted in the literature). In our analysis we have taken into account the undetected population of GRBs through the correction of the observed variables with the Efron \& Petrosian (1992) method.

In Figure 1 (middle and left panels) we then report on the inferred initial magnetic fields (at the neutron star pole) and spin period distributions derived from modeling the plateau luminosities and durations with a GRB-magnetar model (Zhang \& Mészáros 2001), namely:

$$
\begin{aligned}
B_{0 p, 15}^{2} & \simeq 4.2025 I_{45}^{2} R_{6}^{-6}\left[L_{\mathrm{sd}, 49} * \epsilon /(1-\cos \theta)\right]^{-1} \tau_{\mathrm{sd}, 3}^{-2} \\
P_{0,-3}^{2} & \simeq 2.05 I_{45}\left[L_{\mathrm{sd}, 49} * \epsilon /(1-\cos \theta)\right]^{-1} \tau_{\mathrm{sd}, 3}^{-1},
\end{aligned}
$$

where $\epsilon$ is the conversion efficiency of extracting rotational energy from the newly born pulsar, and $\theta$ is the beaming angle. We have studied the dependence of the derived initial $B_{0}$ and $P_{0}$ distributions on these two unknown quantities. Lowering the efficiency factor results in a net shift of the resulting $B$-fields and periods toward lower values (with several GRBs requiring unphysically low values of the birth rotational period; see e.g., Figure 1 right panel). On the other hand, assuming an extremely beamed emission has the opposite effect, shifting all inferred values toward longer spin periods but unreasonably high magnetic fields (see also below). By studying the set of parameters that better reproduce the luminosity-time correlation, Rowlinson et al. (2014) propose a range for $\epsilon /(1-\cos \theta) \simeq 2-4$; this range leads to very high initial magnetic fields. For our purposes, we adopt the less problematic case $\epsilon /(1-\cos \theta)=1$, but our conclusions will be unchanged if we assume larger values.

\footnotetext{
11 http://heasarc.nasa.gov/xanadu/xspec/manual/
} 

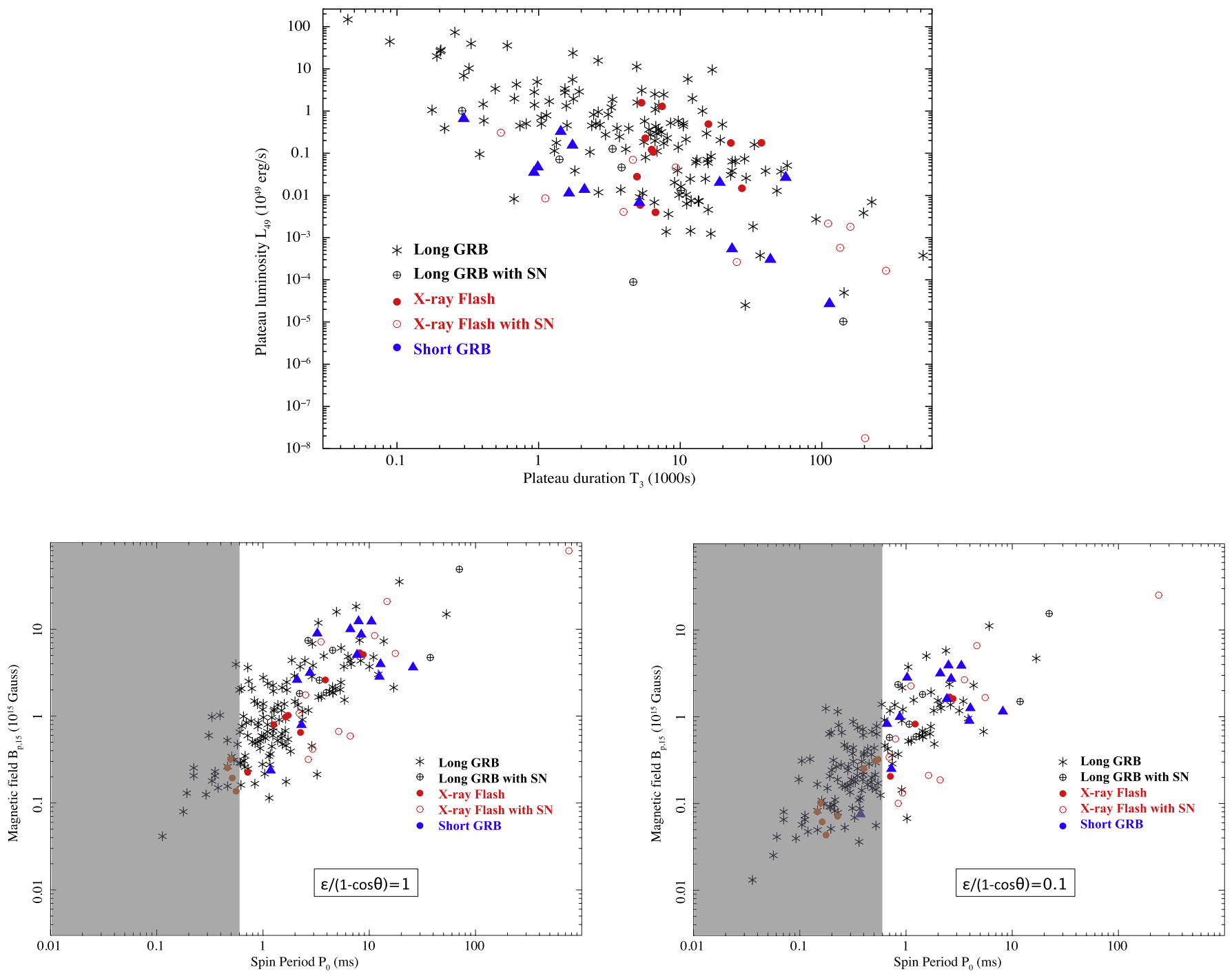

Figure 1. Top panel: the rest-frame plateau durations vs. the luminosity $(1-10,000 \mathrm{keV})$ at the end of the plateaus for all the GRBs in the sample (black $=$ Long GRBs, Blue = Short GRBs, and Red = X-ray Flashes). Bottom panels: derived dipolar fields and initial spin period assuming the GRB-magnetar model for two different values of efficiency $(\epsilon)$ vs. opening angle $(\theta)$ relation (see the text for details). The shaded gray area excludes the rotational periods that would exceed the mass shedding limit under any reasonable neutron star EoS assumption.

From the Swift X-ray plateau modeling we can derive a $B_{0}$ distribution for all GRBs, which we have distinguished in different classes. As it can be noted from Figure 1 (i.e., middle panel), there is no evidence for a distinct $B_{0}$ and $P_{0}$ distributions as a function of the GRB class.

The $B_{0}$ field distribution of the resulting magnetars for all GRBs is well fitted by a log-normal distribution centered at $\log B_{0}=15.1$ with a dispersion of $\sigma \simeq 0.55$. Inferred rotational periods at birth range between $\sim 0.1$ and $70 \mathrm{~ms}$ (with a single outlier around $800 \mathrm{~ms}$ ). We note that the fits give many unphysically short spin periods that would exceed the mass shedding limit, which, depending on the neutron star mass, can be placed between 0.6 and $1.5 \mathrm{~ms}$ (see e.g., Goussard et al. 1998).

\section{NEUTRON STAR POPULATION SYNTHESIS SIMULATIONS AND RESULTS}

In Gullón et al. (2015) we have performed a Population Synthesis analysis considering both the radio-pulsar and the thermal X-ray emitting neutron star populations (comprising the magnetars), taking advantage of $2 \mathrm{D}$ numerical simulations of the magneto-thermal neutron star evolution (Viganò et al. 2013). We refer to Gullón et al. $(2014,2015)$ for details on the Monte Carlo simulations used to synthesize the Galactic neutron star populations. This analysis allowed us to derive the best set of parameters ( $B_{0}$ and $P_{0}$ distributions) consistent with both the current pulsar and magnetar $P-\dot{P}$ distributions. The most important result of this work was the discovery that a single log-normal $B$-field distribution function could not explain at the same time the radio pulsars and the magnetars. Either a truncated $\log$-normal $B$ distribution, or a binormal distribution with two distinct populations, were needed. More importantly, in both cases the current lack of detected isolated $\mathrm{X}$-ray pulsars with periods $>12 \mathrm{~s}$ strongly constrains the number of Galactic neutron stars born with $B_{0}>10^{15} \mathrm{G}$.

We begin the simulations with the assumption of two different populations: normal radio-pulsars, and magnetars associated to GRBs. For the radio-pulsar population, we use the best fit parameters corresponding to model $\mathrm{D}$ in Gullón et al. (2015), which successfully fits the radio-pulsar population properties. The initial magnetic field distribution for the 
synthetic magnetars is assumed to be the one consistent with the GRB-magnetar association obtained in Section 2 (a lognormal distribution centered at $\log B_{0}=15.1$ with dispersion $\sigma=0.55)$. For every synthetic magnetar, the initial period $P_{0}$ was forced to be correlated with $B_{0}$ by the formula: $\log P_{0}=-6.2+0.22 \log B_{0}$, being the observed correlation between these quantities intrinsic, and encompassing all different kind of GRBs (see Figure 1 and Dainotti et al. 2013b; Rowlinson et al. 2014). We stress that the particular choice of $P_{0}$ is completely irrelevant for our results, since the high average magnetic field of the population makes them spindown very fast to reach higher periods. Assuming an initial period of 1 or $10 \mathrm{~ms}$ makes no difference for the results discussed below.

The only parameter that still needs to be fixed is the relative normalization of the number of radio pulsars and GRBmagnetars, the latter being expected to be proportional to the product of the GRB rate $\left(\rho_{\mathrm{GRB}}\right)$ at $z=0$, and the fraction of GRBs expected to leave as a remnant compact object a "stable" magnetar $\left(f_{\text {mag }}\right.$; i.e., that survives subsequent collapse to a black hole). Given the large uncertainties in these two quantities, and the differences related to the different GRB types, we first run simulations and derive probabilities as a function of a general $\rho_{\mathrm{GRB}} * f_{\mathrm{mag}} \equiv N_{\mathrm{gen}}$ : namely the number of "stable" magnetars that were formed via a GRB in the Milky Way in the past million years, regardless of the GRB type (this is allowed by the fact that all types have a similar $B_{0}$ distribution; see Figure 1). We then discuss differences in our conclusions for different GRB types in Section 4. Note that there is no GRB beaming effect involved in the detectability of the remnant as an X-ray pulsar, so there could be unseen GRBs leaving behind a visible magnetar. Initial positions in the Galaxy are drawn from the radial probability distribution of Yusifov \& Küçük (2004) within the Galactic spiral arms. The position of each magnetar is then evolved until its present age, by solving the Newtonian equations of motion under the influence of the smooth Galactic gravitational potential (Carlberg \& Innanen 1987; Kuijken \& Gilmore 1989). The age of each star is randomly uniform in the interval $[0,1]$ Myr. Spatial kick velocities and the inclination angle (between rotational and magnetic axes) are also randomly selected. In order to account for the detections in the X-ray band we assume blackbody emission plus Resonant Compton Scattering, as typical of magnetars' spectra (Rea et al. 2008; Zane et al. 2009). The photoelectric absorption along the line of sight is also considered (see Gullón et al. 2015 for further details).

In Figure 2 we report the results of a typical realization with $N_{\text {gen }}=100$ magnetars (plus the large number of radio-pulsars fitting the radio-pulsar population) by showing their predicted $P-\dot{P}$ and $\log N-\log S$ diagrams for the visible $\mathrm{X}$-ray pulsars at present, compared with the observed sources. We show results with two different cut-offs in absorbed X-ray fluxes, at $10^{-13}$ and $10^{-12} \mathrm{erg} \mathrm{s}^{-1} \mathrm{~cm}^{-2}$. In the left panel, we see that the total number of X-ray pulsars we observe in our Galaxy (after filtering for selection effects) is roughly consistent with the simulated radio pulsars plus 100 GRBmagnetars. This confirms our initial assumption of $\rho_{\mathrm{GRB}} * f_{\mathrm{mag}} \equiv N_{\mathrm{gen}}=100$ in order to explain the currently observed $\sim 20$ magnetars (after selection effects). However, it is clear that their distribution of X-ray fluxes and spin periods are quite different from the observed population. As expected, from the extremely high $B_{0}$ inferred from the GRB plateaus, the simulated GRB magnetars are too bright and too slow compared with the observed magnetars. These discrepancies are even more pronounced if we change the efficiency/ beaming factor $(\epsilon /(1-\cos \theta))$ within the GRB-magnetar scenario, and cannot be mitigated by changing the magnetothermal evolutionary model, or the crustal microphysics assumptions within reasonable values (Pons et al. 2013; Viganò et al. 2013).

Note that if some GRBs not showing a plateau phase still have magnetar central engines, i.e., with lower initial $B$-fields (hence with X-ray plateaus too faint to be detected over the afterglow), this will not change our conclusions, because the initial GRB-magnetar $B$-field distribution will not change systematically to lower values, but the log-normal distribution will only be slightly skewed to include also these putative additional GRB-magnetars with lower $B_{0}$.

Hence, our first result is that our observed population of magnetars cannot be formed via a GRB (regardless of the assumptions on the rates or the different GRB types) because they would have luminosity and period distributions largely inconsistent with the observational data.

We can now estimate the maximum number of "stable" GRB-magnetars in the Milky Way left behind in the past Myr, that is still compatible with the observations.

As shown in Gullón et al. (2015), the number of detectable synthetic magnetars in each realization closely follows a Poissonian distribution with mean value $\lambda=N_{\text {gen }} * p$, where $p$ is the detection probability (note that we assume the GRB rate constant over $1 \mathrm{Myr}$ timescale). We have calculated this probability by performing a large number of runs $(\sim 500$ realizations), and we obtained $p=0.18$ and $p=0.28$ for fluxes $>10^{-12}$ and $>10^{-13}$, respectively.

Since the most constraining observational fact is the lack of $X$-ray pulsars with periods greater than $12 \mathrm{~s}$, we can calculate the probability of not detecting any pulsar with $P>12 \mathrm{~s}$, which is $e^{-\lambda}$. In Figure 3 we plot the no-detection probability of magnetars with spin period $>12 \mathrm{~s}$ as a function of $N_{\text {gen }}$, the number of "stable" magnetars formed in the Milky Way via a GRB in the past Myr. The figure compares the results for two different flux thresholds, $10^{-13} \mathrm{erg} \mathrm{s}^{-1} \mathrm{~cm}^{-2}$ (dashed red) and $10^{-12}$ (solid blue) $\mathrm{erg} \mathrm{s}^{-1} \mathrm{~cm}^{-2}$. These two fluxes roughly encompass the range in which we believe our X-ray sample of detected X-ray pulsar is nearly complete. Thus, assuming our sample is complete above fluxes $>10^{-13} \mathrm{erg} \mathrm{s}^{-1} \mathrm{~cm}^{-2}$, only in one per cent of the simulations we do not find any visible pulsar with $P>12 \mathrm{~s}$, which means that we can establish the upper limit $\rho_{\mathrm{GRB}} * f_{\mathrm{mag}}<16$ with a $99 \%$ confidence level.

The above conclusions are in principle valid for any GRB type leaving behind a "stable" magnetar. However, as we will discuss further in the following section, the upper limit we derived is eventually meaningful only for LGRBs, given that SGRBs are hardly expected to leave any "stable" magnetar, and expected to collapse into a black hole soon after the X-ray plateau phase in most of the cases.

\section{DISCUSSION}

We have performed neutron star population synthesis simulations, to set constraints on the GRB-magnetar scenario by means of the Galactic population of highly magnetized neutron stars. By assuming that the X-ray plateau phases of GRB afterglows are powered by the rotational energy of a newly born, rapidly spinning magnetar central engine, we 

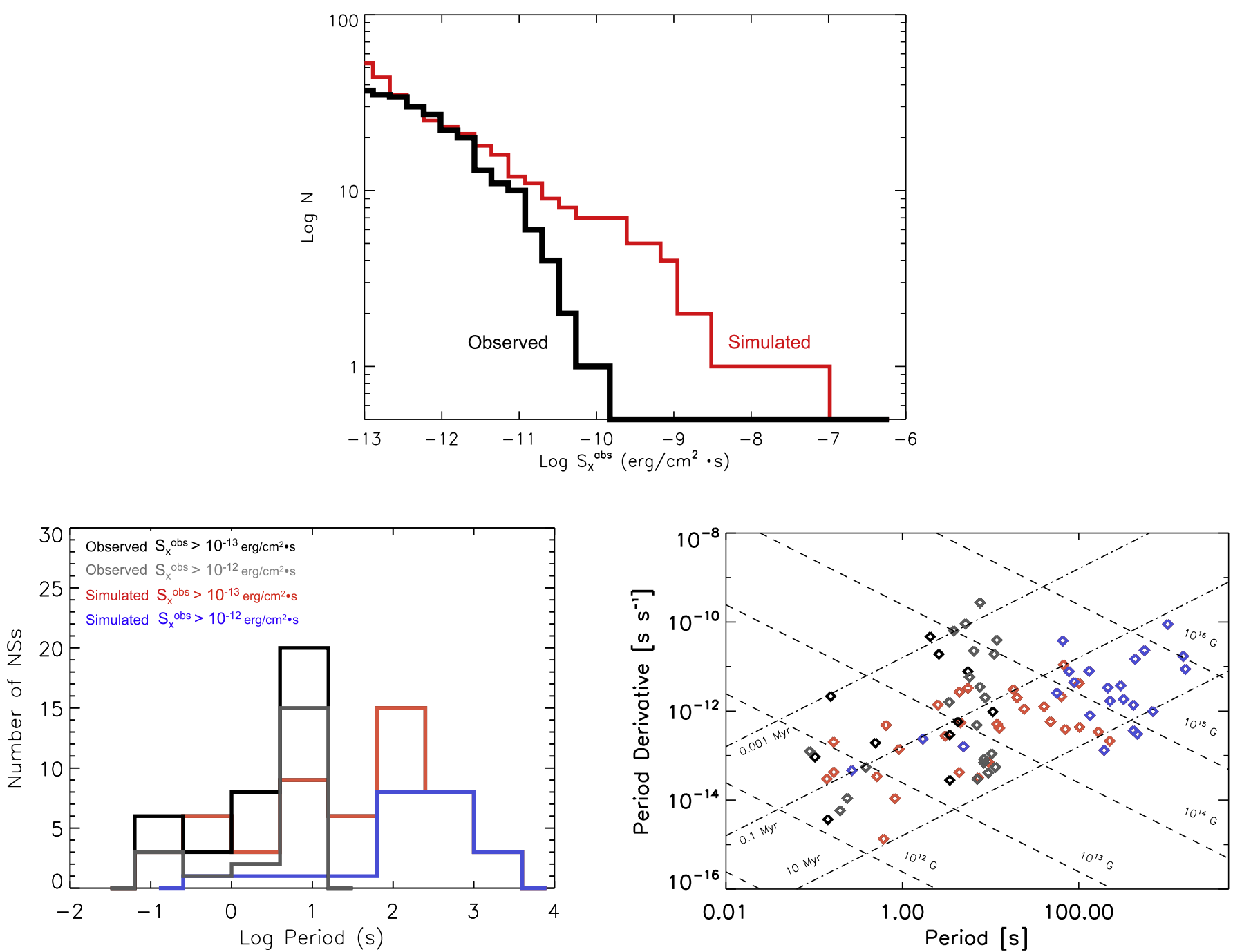

Figure 2. Results of the Population Synthesis Simulations of our X-ray pulsar population plus 100 "stable" magnetars formed via a GRB in the past Myr in our Galaxy. Top panel: $\log N-\log S$ of the simulated sample (red) compared with the observed X-ray fluxes (black). Bottom panels: spin period distribution of the observed $\mathrm{X}$-ray pulsar population compared with the "observable" simulated sample of synthetic GRB-magnetars, and relative $P-\dot{P}$ diagram. In the latter, the black and gray symbols represent the observed objects, while the synthetic sample is displayed in red and blue. Gray and blue dots represent pulsars with X-ray fluxes of $>10^{-12} \mathrm{erg} \mathrm{s}^{-1} \mathrm{~cm}^{-2}$; black and red dots are objects with fluxes $10^{-13}<f_{X}<10^{-12} \mathrm{erg} \mathrm{s}^{-1} \mathrm{~cm}^{-2}$.

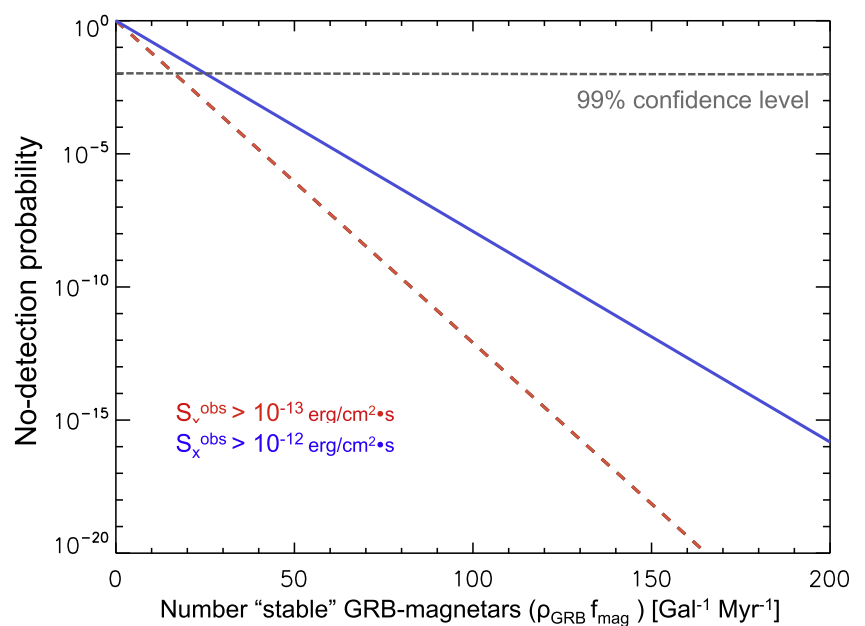

Figure 3. Probability of the no-detection of a synthetic magnetar with a spin period $>12 \mathrm{~s}$ as a function of the injected number of "stable" magnetars for two cuts fluxes: $10^{-13}$ (dashed red) and $10^{-12}$ (solid blue) erg s $\mathrm{em}^{-1}$. The gray line indicates the $99 \%$ confidence level. derived from the Swift GRB sample the resulting initial $B$-field and spin period distribution of such newly born magnetars. Using these distributions, we simulated the properties of a synthetic population of magnetars formed in our Galaxy in the past Myr via a GRB.

We found that, if we assume $\sim 100$ GRBs leaving behind a "stable" magnetar in the past Myr, the number of "observable" objects (considering the predicted properties of such simulated magnetars and all the observational biases) roughly agrees with the number of magnetars we currently observe in the Milky Way ( 20). However, the properties of the simulated sample of GRB-magnetars are inconsistent with what observed: they are too bright, and spin too slowly, with spin periods far exceeding the observed limit of $12 \mathrm{~s}$ for the Galactic pulsar population (see Pons et al. 2013).

This result was not totally unexpected given that, to model the current population of pulsars and magnetars in our Galaxy, it was recently observed that the initial $B$-field distribution should not allow fields in excess of $10^{15} \mathrm{G}$, otherwise the limiting spin period observed in isolated pulsars $(\sim 12 \mathrm{~s})$ cannot be reconciled (Gullón et al. 2015; Popov 2015). The magnetars 
that GRBs need to form to supply spin-down energy to the $\mathrm{X}$-ray plateaus, are "super-magnetars," having initial $B$-fields significantly larger than those extrapolated for our Galactic magnetars.

Given that the number of observable magnetars is reproduced, but their general properties are not, we can safely conclude that assuming the GRB-magnetar scenario in its present formulation, in particular that X-ray plateaus are powered by spin-down energy, our Galactic magnetars (regardless the assumed GRB type or rate at $z=0$ ) should be mostly formed by a distinct formation path than a GRB. Most likely a type of Core-Collapse $\mathrm{SNe}$ different from the Type Ib/ c connected to LGRBs. In this contest, this would also mean that GRB-like SNe should systematically produced stronger magnetic fields in the proto-magnetar than other CC-SNe. ${ }^{12}$

\subsection{General Estimates of the Fraction of Expected Stable Magnetars $\left(f_{\mathrm{mag}}\right)$, and GRB Rates at $\mathrm{z}=0$}

To put our simulations in contest, we discuss here current estimates of the local GRB rates, and of the probability for a magnetar born associated to a GRB to survive or collapse to a black hole after the X-ray plateau phase. Note that both these quantities are extremely uncertain.

If we proceed observationally to derive $f_{\text {mag }}$, within the GRB-magnetar model, we can assume that if an X-ray plateau is observed the GRB formed a magnetar. From the Swift GRB reanalysis we derived that, for LGRBs, in $70 \%$ of the cases we can reasonably fit a plateau phase (137 cases over 195), for SGRBs, a plateau improves the fit in $23 \%$ of the cases, and for XRFs in $100 \%$. We then assume this percentage as the minimum percentage of GRBs having a magnetar engine powering the plateaus (in the others the plateau could had been missed or too faint).

Subsequently, we assume to zeroth order that if there is no collapse onto a black hole (i.e., due to residual accretion onto the newly formed magnetar), at the end of the X-ray plateau there is no sharp decay in time, and the afterglow decays as $t^{-\alpha}$, with $\alpha \leqslant 2$. To estimate the fraction of magnetars that collapse, we have counted in how many cases we found a subsequent $t^{-\alpha}$ decay with $\alpha>2$. We find that such steep decay after the X-ray plateau is detected in 14 LGRBs, among the 137 with an X-ray plateau. We can then roughly estimate that the fraction of LGRBs leaving a "stable" magnetar is $f_{\text {mag }} \sim 0.63$.

Regarding the determination of the LGRB rates at $z=0$, two main approaches have been discussed in the literature (in addition to the limits inferred via radio afterglow constraints; Perna \& Loeb 1998). The first approach derives the local LGRB rate from the GRB association with $\mathrm{SN}$ Type $\mathrm{Ib} / \mathrm{c}$. Radio and optical SN surveys suggest that $\sim 25 \%$ of all CCsupernovae are Type $\mathrm{Ib} / \mathrm{c}$, but only 3\%-10\% of those are related to LGRBs (Berger et al. 2003; Soderberg et al. 2006, 2010; Lien et al. 2014). In the local universe this type of SNe have a rate of $\rho_{\mathrm{SN}-\mathrm{Ib} / \mathrm{c}}=1.7 \times 10^{4} \mathrm{Gpc}^{-3} \mathrm{year}^{-1}$ (Cappellaro et al. 1999; Soderberg et al. 2010). With one galaxy in $100 \mathrm{Mpc}^{3}$ (or equivalently, with the Milky Way volume of about $10^{-7} \mathrm{Gpc}^{3}$; Panter et al. 2007) this

\footnotetext{
12 Unfortunately assessing whether this is or is not the case is currently beyond the capabilities of current simulations of magnetic field formation in protoneutron stars (and certainly far from the aim of this work).
}

results in $~ 50-170$ GRB-SN Ib/c events within the last million years.

A different approach relies on a direct inversion of the redshift-luminosity distribution of the observed LGRBs to infer their local rate. This method needs to impose a low-luminosity cutoff to avoid divergences (see e.g., discussion in Guetta et al. 2004). A comprehensive study with Swift GRBs up to 2010 was performed by Wanderman \& Piran (2010). With a low- $L$ cutoff of $L>L_{50} \equiv 10^{50} \mathrm{erg} \mathrm{s}^{-1}$, they inferred a local rate of $\rho_{\mathrm{LGRB}\left(L>L_{50}\right)}=(1.3 \pm 0.6) f_{b}^{-1}\left(\mathrm{Gpc}^{-3} \mathrm{year}^{-1}\right)$. Correcting for a beaming factor of about $f_{b}^{-1}=70$ (see Guetta et al. 2005; Fong et al. 2012), we expect $\sim 9 \mathrm{LGRB}_{L>50}$ events in $1 \mathrm{Myr}$. We caveat here that there might be a metallicity dependence in extrapolating this GRB rate at $z=0$ (in particular Milky Waylike galaxies seem not to be the preferred hosts for LGRBs; see e.g., Robertson \& Ellis 2012; Salvaterra et al. 2012; Trenti et al. 2013, 2015). However, while some evidence points toward a preference of LGRBs for low-metallicity hosts (e.g., Modjaz et al. 2008; Graham \& Fruchter 2013), some outliers have also been discovered (Savaglio et al. 2012; Levesque 2014). The uncertain dependence of the GRB rate on metallicity and star formation, as well as on redshift, only contributes to increase the uncertainties of the local LGRB rate determination (Jimenez \& Piran 2013; Dainotti et al. 2015a). For the above reasons we do not enter in the metallicity/redshift/star formation rate dependence discussion, especially because it is not so relevant for the work presented here.

As Wanderman \& Piran (2010) discuss in their Section 6.2, there are several low-luminosity $\mathrm{LGRBs}_{L<50}$ that are not taken into account in their estimated rate. Given their faint nature, LGRBs with $L<L_{50}$ could have a rate much larger than for brighter LGRBs, but at this time it remains even more uncertain. Current estimates state that they should be roughly 10 times more numerous than the $\mathrm{LGRBs}_{L>50}$ (Soderberg et al. 2006, 2010), and have very low beaming factors. Guetta \& Della Valle (2007) attempted to estimate their local rates on the basis of the few known events, and inferred $\sim 380_{-225}^{+620}$ $\left(\mathrm{Gpc}^{-3}\right.$ year $\left.^{-1}\right)$, which would result in about 38 low-luminosity LGRB events in the past Myr (again with large errors). This is consistent with a similar estimate found by Liang et al. (2007), assessing the rate of the low-luminosity LGRBs as $\sim 0.7 \%$ of all Type $\mathrm{Ib} / \mathrm{c} \mathrm{SN}$.

Summarizing, the different approaches estimate that the total (very uncertain), beaming corrected, LGRBs rate at $z=0$ should range within $\sim 50-170$ (considering also the low luminosity ones) in our Galaxy in the past Myr, depending on the different approaches in the literature.

For SGRBs, Wanderman \& Piran (2015) derived $\rho_{\mathrm{SGRB}}=(4 \pm 2) f_{b}^{-1}\left(\mathrm{Gpc}^{-3}\right.$ year $\left.^{-1}\right)$, where $f_{b}^{-1}$ is the GRB beaming factor. Assuming $f_{b}^{-1}=30$ (see Fong et al. 2012), we then expect $\sim 12$ SGRB events in our Galaxy in the past Myr. The estimate of $f_{\text {mag }}$ for SGRBs is even more difficult than for LGRBs. Observationally, this is very much limited by the smaller sample to be meaningful. On the other hand, theoretically, while it has been demonstrated via general relativistic, magnetohydrodynamical simulations that the formation of a stable neutron star from the merger of two small neutron stars $\left(\sim 1.2 M_{\odot}\right)$ is possible (Giacomazzo \& Perna 2013; Dall'Osso et al. 2015), the formation rate depends on the rate at which the small-mass neutron stars are formed at birth, as well as on the neutron star equation of state (which 
determines the maximum mass of the resulting magnetar), and on the magnitude of the subsequent rate of accretion.

All in all, since the statistics of neutron star masses in binaries are still too small to draw quantitative estimates, and the local, galactic SGRB rates are smaller than those of LGRBs anyways, we have adopted the conservative assumption that the possible contribution from SGRBs to the observed galactic magnetar population is negligible (note also that Galactic magnetars are mostly located in the Galactic plane and in massive star clusters, unlike what would be expected for the remnants of a compact merger). Hence, even if our results are not dependent on the GRB type, but require only such GRB to leave a "stable" magnetar behind, eventually our conclusions and constraints are meaningful only for the LGRB population.

\subsection{Constraints on LGRBs}

With our simulations we have also estimated the probability of non-detecting a GRB-formed magnetar in our current population as a function of the number of "stable" magnetars that a GRB, mainly LGRBs, have left in the galaxy in the last Myr, namely $\rho_{\mathrm{GRB}} * f_{\mathrm{mag}}$. We find that, in order to reconcile at a $99 \%$ confidence level the non-detection of a GRB-magnetar compact remnant in our Galaxy (meaning non-detecting any magnetar with $P>12 \mathrm{~s}$ ), the quantity $\rho_{\mathrm{GRB}} * f_{\mathrm{mag}}$ should not exceed $\leqslant 16 \mathrm{Gal}^{-1} \mathrm{Myr}^{-1}$. This number depends mainly on the completeness of the X-ray sample of observed neutron stars, hence it can be revised further, and become more stringent, with the advent of new deep X-ray surveys such as eROSITA (Merloni et al. 2012).

Extrapolating current LGRB rate estimates, we derived rough values of $\rho_{\mathrm{LGRB}} \sim 50-170 \mathrm{Gal}^{-1} \mathrm{Myr}^{-1}$, and $f_{\text {mag }} \sim 0.63$ (from fitting the Swift data), that result in $\rho_{\text {LGRB }} * f_{\text {mag }} \sim 30-110$. This is somewhat larger (although with large uncertainties) than the maximum allowed number of GRB "super-magnetars" in our Galaxy $(<16$ at $99 \%$ confidence level).

\section{CONCLUSIONS}

Our results show that the initial $B$-field distribution needed to explain the GRB X-ray plateaus in terms of a fast spinning magnetar does not reconcile the properties of these GRBmagnetars with our Galactic magnetar population, even using the most favorable choices of efficiency/beaming factors. We should then allow the existence of magnetars and "supermagnetars," with two different progenitors and formation path, and different magnetic field formation efficiency.

Even though the large uncertainties in the GRB rates at $z=0$, in the metallicity and star formation rate dependences, and in the fraction of neutron stars collapsing to a black hole, do not allow anyhow to rule out the GRB-magnetar model on the basis of the observed Galactic population, several finetunings are needed to maintain the model in its present form, and keeping the interpretation that X-ray plateaus are necessarily due to spin-down energy (i.e., we should allow some progenitors or environments to create systematically more magnetic stellar remnants than others).

If those stable GRB-formed "super-magnetars" indeed exist, their current non-detection in our Galaxy can be used to put limits on $\rho_{\text {LGRB }} * f_{\text {mag }}$, that will get possibly more and more constraining by means of future deep X-ray surveys.
N.R. thanks A. Rowlinson, B. Metzger, R. Margutti, B. Zhang, S. Dall'Osso, A. Soderberg, R. Wijers, A. MacFayden, M. Modjaz, S. Campana, P. D’ Avanzo, M. G. Bernardini, and L. Rezzolla for useful discussions and/or comments on the manuscript, and the referee for the careful reading. N.R. is supported by an NWO Vidi Grant, and kindly acknowledges Harvard ITC, NYU, Stony Brook University and the MIAPP institute in Garching, for the hospitality during the preparation of this work. N.R. and D.F.T. are also supported by grants AYA2012-39303 and SGR2014-1073. M.G. is supported by the fellowship BES-2011-049123. M.G., J.A.P. and J.A.M. acknowledge support by grants AYA2013-42184-P and Prometeu/2014/69. R.P. acknowledges support from NSF grant No. AST 1009396. M.G.D. is supported by FP7PEOPLE-2013-IOF under the grant agreement number 626267, and thanks the ITHES group and the Astrophysical Big Bang Laboratory for fruitful discussions. This work is partially supported by the European COST Action MP1304 (NewCOMPSTAR).

\section{REFERENCES}

Avni, Y. 1976, ApJ, 210, 642

Berger, E., Kulkarni, S. R., Frail, D. A., \& Soderberg, A. M. 2003, ApJ, 599, 408

Bernardini, M. G., Margutti, R., Mao, J., Zaninoni, E., \& Chincarini, G. 2012, A\&A, 539, A3

Cappellaro, E., Evans, R., \& Turatto, M. 1999, A\&A, 351, 459

Carlberg, R. G., \& Innanen, K. A. 1987, AJ, 94, 666

Dainotti, M. G., Cardone, V. F., \& Capozziello, S. 2008, MNRAS, 391, 79

Dainotti, M. G., Cardone, S., Capozziello, V. F., Willingale, R. S., \& Ostrowski, M. 2011a, ApJ, 730, 135

Dainotti, M. G., Cardone, V. F., Piedipalumbo, E., \& Capozziello, S. 2013a, MNRAS, 436, 82

Dainotti, M. G., Del Vecchio, R., Shigehiro, N., \& Capozziello, S. 2015a, ApJ, 800,31

Dainotti, M. G., Ostrowski, M., \& Willingale, R. 2011b, MNRAS, 418, 2202 Dainotti, M. G., Petrosian, V., Singal, J., \& Ostrowski, M. 2013b, ApJ, 774,157

Dainotti, M. G., Petrosian, V., Willingale, R., et al. 2015b, MNRAS, 451, 3898

Dainotti, M. G., Willingale, R., Capozziello, S., Fabrizio Cardone, V., \& Ostrowski, M. 2010, ApJL, 722, L215

Dall'Osso, S., Stratta, G., Guetta, D., et al. 2011, A\&A, 526, A121

Dall'Osso, S., Giacomazzo, B., Perna, R., \& Stella, L. 2015, ApJ, 798, 25

Duffell, P. C., \& MacFadyen, A. I. 2015, ApJ, 806, 205

Duncan, R. C., \& Thompson, C. 1992, ApJ, 392, L9

Evans, P. A., Beardmore, A. P., Page, K. L., et al. 2009, MNRAS, 397, 1177 Efron, B., \& Petrosian, V. 1992, ApJ, 399, 345

Fong, W., Berger, E., Margutti, R., et al. 2012, ApJ, 756, 189

Giacomazzo, B., \& Perna, R. 2013, ApJ, 771, 26

Goussard, J.-O., Haensel, P., \& Zdunik, J. L. 1998, A\&A, 330, 1005

Graham, J. F., \& Fruchter, A. S. 2013, ApJ, 774, 119

Guetta, D., \& Della Valle, M. 2007, ApJL, 657, L73

Guetta, D., Perna, R., Stella, L., \& Vietri, M. 2004, ApJ, 615, 73

Guetta, D., Piran, T., \& Waxman, E. 2005, ApJ, 619, 412

Gullón, M., Miralles, J. A., Viganò, D., \& Pons, J. A. 2014, MNRAS, 443, 1891

Gullón, M., Pons, J. A., Miralles, J. A., et al. 2015, MNRAS, 454, 615

Jimenez, R., \& Piran, T. 2013, ApJ, 773, 126

Kuijken, K., \& Gilmore, G. 1989, MNRAS, 239, 651

Kumar, P., Narayan, R., \& Johnson, J. L. 2008, MNRAS, 388, 1729

Levesque, E. M. 2014, PASP, 126, 1

Liang, E., Zhang, B., Virgili, F., \& Dai, Z. G. 2007, ApJ, 662, 1111

Lien, A., Sakamoto, T., Gehrels, N., et al. 2014, ApJ, 783, 24

Lü, H.-J., \& Zhang, B. 2014, ApJ, 785, 74

Lyons, N., O'Brien, P. T., Zhang, B., et al. 2010, MNRAS, 402, 705

Margutti, R., Zaninoni, E., Bernardini, M. G., et al. 2013, MNRAS, 428, 729

Mereghetti, S. 2008, A\&ARv, 15, 225

Merloni, A., Predehl, P., Becker, W., et al. 2012, (arXiv:1209.3114)

Mészáros, P., \& Rees, M. J. 1997, ApJ, 476, 232

Metzger, B. D., Giannios, D., Thompson, T. A., Bucciantini, N., \& Quataert, E. 2011, MNRAS, 413, 2031 
Modjaz, M., et al. 2008, AJ, 135, 1136

Nousek, J. A., Kouveliotou, C., Grupe, D., et al. 2006, ApJ, 642, 389

O'Brien, P. T., Willingale, R., Osborne, J., et al. 2006, ApJ, 647, 1213

Panter, B., Jimenez, R., Heavens, A. F., \& Charlot, S. 2007, MNRAS, 378,1550

Perna, R., \& Loeb, A. 1998, ApJ, 509, 85

Popov, S. B. 2015, e-print, (arXiv:1507.06127)

Pons, J. A., Viganò, D., \& Rea, N. 2013, NatPh, 9, 431

Rea, N., Zane, S., Turolla, R., Lyutikov, M., \& Götz, D. 2008, ApJ, 686, 1245

Rea, N., \& Esposito, P. 2011, in Astrophysics and space Science Proc., HighEnergy Emission from Pulsars and their Systems, ed. D. F. Torres \& N. Rea (Berlin: Springer), 247

Robertson, B. E., \& Ellis, R. S. 2012, ApJ, 744, 95

Rowlinson, A., O'Brien, P. T., Tanvir, N. R., et al. 2010, MNRAS, 409, 531 Rowlinson, A., O'Brien, P. T., Metzger, B. D., Tanvir, N. R., \& Levan, A. J. 2013, MNRAS, 430, 1061

Rowlinson, A., Gompertz, B. P., Dainotti, M., et al. 2014, MNRAS, 443, 1779

Salvaterra, R., Campana, S., Vergani, S. D., et al. 2012, ApJ, 749, 68
Sari, R., Piran, T., \& Narayan, R. 1998, ApJL, 497, L17

Savaglio, S., Rau, A., Greiner, J., et al. 2012, MNRAS, 420, 627

Soderberg, A. M., Chakraborti, S., Pignata, G., et al. 2010, Natur, 463, 513

Soderberg, A. M., Nakar, E., Berger, E., \& Kulkarni, S. R. 2006, ApJ, 638, 930

Trenti, M., Perna, R., \& Jimenez, R. 2015, ApJ, 802, 103

Trenti, M., Perna, R., \& Tacchella, S. 2013, ApJ, 773L, 22

Troja, E., Cusumano, G., O’Brien, P. T., et al. 2007, ApJ, 665, 599

Usov, V. V. 1992, Natur, 357, 472

Viganò, D., Rea, N., Pons, J. A., et al. 2013, MNRAS, 434, 123

Wanderman, D., \& Piran, T. 2010, MNRAS, 406, 1944

Wanderman, D., \& Piran, T. 2015, MNRAS, 448, 3026

Willingale, R., O'Brien, P. T., Osborne, J. P., et al. 2007, ApJ, 662, 1093

Xiao, L., \& Schaefer, B. E. 2009, ApJ, 707, 387

Yusifov, I., \& Kuumlçük, I. 2004, A\&A, 422, 545

Zane, S., Rea, N., Turolla, R., \& Nobili, L. 2009, MNRAS, 398, 1403

Zhang, B., Fan, Y. Z., Dyks, J., et al. 2006, ApJ, 642, 354

Zhang, B., \& Mészáros, P. 2001, ApJL, 552, L35 